\title{
Spin polarization effects in micro black hole evaporation
}

\author{
Antonino Flachi. * Misao Sasaki * $^{\text {A }}$ and Takahiro Tanaka \\ Yukawa Institute for Theoretical Physics, Kyoto University, Kyoto 606-8502, Japan
}

\begin{abstract}
We consider the evaporation of rotating micro black holes produced in highly energetic particle collisions, taking into account the polarization due to the coupling between the spin of the emitted particles and the angular momentum of the black hole. The effect of rotation shows up in the helicity dependent angular distribution significantly. By using this effect, there is a possibility to determine the axis of rotation for each black hole formed, suggesting a way to improve the statistics. Deviation from thermal spectrum is also a signature of rotation. This deviation is due to the fact that rapidly rotating holes have an effective temperature $T_{\text {eff }}$ significantly higher than the Hawking temperature $T_{H}$. The deformation of the spectral shape becomes evident only for very rapidly rotating cases. We show that, since the spectrum follows a blackbody profile with an effective temperature, it is difficult to determine both the number of extra-dimensions and the rotation parameter from the energy spectrum alone. We argue that the helicity dependent angular distribution may provide a way to resolve this degeneracy. We illustrate the above results for the case of fermions.
\end{abstract}

Introduction. Within the context of TeV-scale gravity [1, 2, 3], the possibility that colliders or cosmic ray facilities may observe micro black holes has attracted enormous attention [5, 6, 7, 8, 9]. A close look at the limits on the fundamental Planck scale shows that a window of about $5 \mathrm{TeV}$ is still open for the LHC to observe such exotic events [10], while the window is much wider for cosmic rays. Micro black holes with even higher energies could be produced from the collision of a cosmic ray with an atmospheric nucleon, a dark matter particle, or another cosmic ray (Ref. 11] gives some up-to-date estimates).

In this paper, we consider micro black holes resulting from the collision of two particles at energies much higher than the higher dimensional Planck mass $M_{P}$. We have in mind models with $M_{P}$ of order of a few $\mathrm{TeV}$ and the standard model confined on a 3-brane, embedded in a $(4+n)$-dimensional bulk. These black holes have horizon radius smaller than the size of the extra dimensions, and are expected to follow balding, spin-down, Schwarzschild, and Planck phases. Micro black hole formation has been studied both analytically 12 and numerically [13, 14], and their evaporation has also been the subject of considerable attention (See Refs. 10, 16 for review). Previous work suggests that micro black holes will emit mostly brane modes [17, 18], and the deviations from the blackbody spectrum have been investigated using numerical and semi-analytical methods [19, 20, 21, 22, 23, 24, 25, 26].

Radiation. In this paper we analyze the fermion emission from spinning evaporating micro black holes. Assuming that the black hole horizon is significantly smaller than the extra dimensions, we approximate it by a vac-

*Electronic address: flachi AT'yukawa.kyoto-u.ac.jp

${ }^{\dagger}$ Electronic address: misao AT yukawa.kyoto-u.ac.jp

${ }^{\ddagger}$ Electronic address: tanaka AT’yukawa.kyoto-u.ac.jp uum higher dimensional Kerr [27]:

$$
\begin{aligned}
d s^{2}= & \left(1-\frac{M}{\Sigma r^{n-1}}\right) d t^{2}+\frac{2 a M \sin ^{2} \theta}{\Sigma r^{n-1}} d t d \varphi-\frac{\Sigma}{\Delta} d r^{2} \\
& -\Sigma d \theta^{2}-\left(r^{2}+a^{2}+\frac{a^{2} M \sin ^{2} \theta}{\Sigma r^{n-1}}\right) \sin ^{2} \theta d \varphi^{2} \\
& -r^{2} \cos ^{2} \theta d \Omega_{n}^{2},
\end{aligned}
$$

where $\Delta \equiv r^{2}+a^{2}-M r^{1-n}$ and $\Sigma \equiv r^{2}+a^{2} \cos ^{2} \theta . M_{P}$ is normalized to one. Since we are interested in the visible brane modes, the background spacetime will be given by the projection of the above metric on the brane.

Massless fermions emitted by the black hole are described by the Dirac equation:

$$
e_{a}^{\mu} \gamma^{a}\left(\partial_{\mu}+\Gamma_{\mu}\right) \psi=0
$$

where $\psi$ is the Dirac spinor wave function, $e_{a}^{\mu}$ a set of tetrads, $\Gamma_{\mu}$ the spin-affine connections determined by $\Gamma_{\mu}=\gamma^{a} \gamma^{b} \omega_{a b \mu} / 4$, with $\omega_{a b \mu}$ being the Ricci rotation coefficients. The matrices $\gamma^{\mu}=e_{a}^{\mu} \gamma^{a}$ are chosen to satisfy the relation $\gamma^{\mu} \gamma^{\nu}+\gamma^{\nu} \gamma^{\mu}=g^{\mu \nu}$, with $g^{\mu \nu}$ being the metric on the brane.

The Dirac equation for massless fermions on a Kerr background has been studied extensively in four and higher dimensions [20, 25, 26, 28, 29, 30, 31]. Here we briefly describe the calculation, closely following the approach of Ref. [28]. Due to the symmetries of the Kerr spacetime, the spinor wave function factorizes as

$$
\psi=\mathcal{N} e^{i(m \varphi-\omega t)}\left(\begin{array}{c}
\vec{\phi} \\
\pm \vec{\phi}
\end{array}\right)
$$

where the + and - signs refer to negative and positive helicities, respectively. We illustrate the results for the case of negative helicity. The positive helicity case can be obtained by a trivial chirality transformation. The field $\vec{\phi}$ takes the form

$$
\vec{\phi}=\left(\begin{array}{c}
R_{-}(r) S_{-}(\theta) \\
R_{+}(r) S_{+}(\theta)
\end{array}\right)
$$



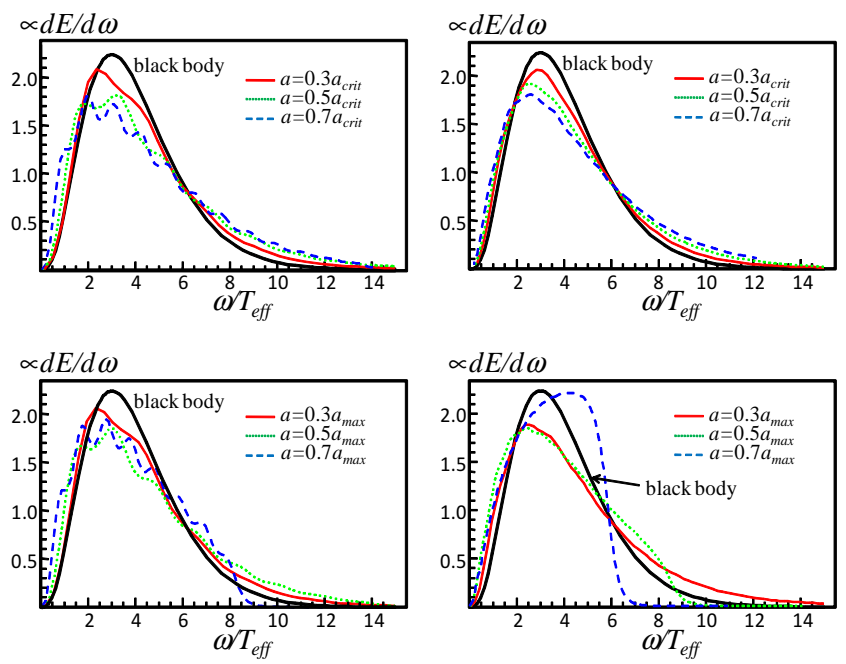

FIG. 1: The normalized energy spectrum of the emitted fermions. The horizontal axis is rescaled by the effective temperature determined by fitting the data by a black body profile. The overall amplitude is also normalized since the absolute magnitude is not observable. The upper and the lower panels are the plots for $n=2$ and $n=4$, respectively. The rotation parameter $a$ is set to $30 \%, 50 \%$ and $70 \%$ of $a_{\text {crit }}$ (left) and $a_{\max }$ (right).

and the normalization factor is $\mathcal{N}^{-1}=\Delta^{1 / 4}(r+$ $i a \cos \theta)^{1 / 2} \sin ^{1 / 2} \theta$. The angular and radial modes obey

$$
\begin{aligned}
\left(\frac{d}{d \theta} \pm \omega a \sin \theta \mp \frac{m}{\sin \theta}\right) S_{\mp}(\theta) & = \pm \kappa S_{ \pm}(\theta), \\
\left(\frac{d}{d r} \mp \frac{i}{\Delta}\left(\omega\left(r^{2}+a^{2}\right)-m a\right)\right) R_{\mp}(r) & =\kappa \Delta^{-1 / 2} R_{ \pm}(r),
\end{aligned}
$$

where $\kappa$ is a separation constant. Supplemented with regularity conditions at $\theta=0$ and $\pi$, the set of angular equations provides an eigenvalue problem, which determines $\kappa$ [30]. In order to compute the particle flux, we need the solution to the radial equation supplemented by ingoing boundary conditions at the horizon

$$
R_{-} \sim 0, \quad R_{+} \sim e^{-i \tilde{\omega} r_{*}}, \text { for } r_{*} \rightarrow-\infty
$$

where $\tilde{\omega}=\omega-m a /\left(r_{h}^{2}+a^{2}\right)$, and $r_{*}$ is defined by $d r_{*} / d r=\left(r^{2}+a^{2}\right) / \Delta$. The number of particles emitted, for fixed frequency $\omega$, is distributed according to the Hawking radiation formula. For negative helicity modes, the angular distribution reads:

$$
\frac{d N}{d \omega d \cos \theta}=\frac{1}{2 \pi \sin \theta} \sum_{l, m}\left|S_{-}(\theta)\right|^{2} \frac{\sigma_{l, m}}{e^{\tilde{\omega} / T_{H}}+1},
$$

where $T_{H}=\frac{1}{4 \pi r_{h}} \frac{(n+1) r_{h}^{2}+(n-1) a^{2}}{r_{h}^{2}+a^{2}}$ is the Hawking temperature, and the grey-body factor $\sigma_{l, m}$ is the squared amplitude of the transmission coefficient of an incoming wave from $r=\infty$ (see Ref. [31]).
Results. The initial angular momentum of the produced black holes $J=2 a M /(n+2)$ is restricted by requiring the impact parameter $b=J / M$ to be smaller than the horizon radius $r_{h}$, determined by $\Delta\left(r_{h}\right)=0$. Then, the maximum value of the rotation parameter $a$ turns out to be $a_{\max }=\frac{n+2}{2} r_{h}[20]$. The upper bound on $J$ might be even lower for $n \geq 2$. In fact, there exists a critical value for $a, a_{\text {crit }} \equiv(n+1)(n-1)^{-1} r_{h}^{2}$, where $\left|\partial\left(T, \Omega_{h}\right) / \partial(M, J)\right|$ vanishes. If the same argument as in the case of black branes applies, black holes with $a>a_{\text {crit }}$ suffer from the Gregory Laflamme instability (See also Ref. [32]). Then, $a_{c r i t}$ represents the maximal value below which the higher dimensional Kerr solution is adequate. The value at which the dynamical instability is expected to set in may be slightly different from $a_{c r i t}$, which only represents an indicative estimate of the critical value. Interestingly $a_{c r i t}<a_{\max }$ (for $n=2,3,4$ extra dimensions, $a_{\text {crit }}=1.09,1.07,1.06$, whereas $\left.a_{\max }=1.25,1.89,2.46\right)$. Although it is widely believed that a dynamical instability exists, the value of $a_{c r i t}$ obtained above is only heuristic. Thus, we consider two possible cases: the maximal value allowed for $a$ is $a_{\text {crit }}$ or $a_{\max }$. A set of representative values for the parameter $a$ is chosen as $a / a_{\max }=0.3,0.5,0.7$, and $a / a_{\text {crit }}=0.3,0.5,0.7 . \quad M$ is set to unity. Another natural choice to present the results would be to use the impact parameter $b$. In our case, we use the ratios $a / a_{\text {crit }}$ and $a / a_{\max }$, which correspond respectively to $b / b_{c r i t}$ and $b / b_{\max }$, where $b_{\text {crit }}$ and $b_{\max }$ are the values for the impact paramter corresponding to the critical and maximal cases respectively.

Having fixed $a$ in the above way, we compute the energy spectrum, shown in Fig. 1. We normalize the horizontal axis by using an effective temperature $T_{\text {eff }}$ determined by fitting the data by a blackbody spectrum profile. The effective temperature $T_{\text {eff }}$ is much higher than the Hawking temperature as shown in Fig. 2 However, the spectral shape is not so different from the thermal one except for the cases with $a \approx a_{\max }$ (Fig. (1). In previous work, [25, 26], the enhancement of emission at large frequencies is reported. However, the deviation from a blackbody spectrum was not quantified.

We find that the renormalized spectra are enhanced for both lower and higher frequencies compared with the black body spectrum at $T=T_{\text {eff }}$ (thick line). Except for very large values of $a$, we have shown that the obtained spectra can be fit well by superpositions of black body profiles with width of about $2 \Omega_{H} \times T_{\text {eff }}$. The intuitive reason for the enhancement of the effective temperature is that the motion of the hypothetical emitting surface on the rotating black hole, relative to observers at infinity, causes an additional blueshift factor (which varies from place to place). This is because corotating emitted particles encounter less suppression from the statistical factor. This can be made precise by closely inspecting the combined behavior of the greybody and statistical factors. The dominant contribution to the spectrum comes from the $l=m$ modes and for larger values of the rotation 

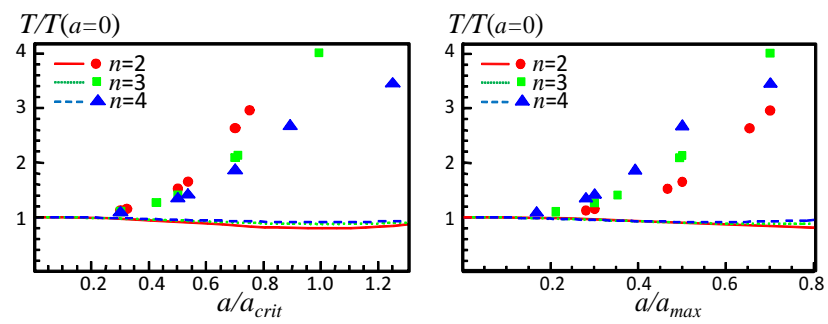

FIG. 2: The effective temperature normalized by the temperature at $a=0$ versus the rotation parameter $a$ normalized by $a_{\text {crit }}$ (left) and $a_{\max }$ (right). The curves represent the Hawking temperature.

the contribution to the spectrum from such modes, with large $l$, is non negligible.

However, because of the change in the temperature and the rotation parameter during the evaporation, the broadening of the spectrum due to the rotation will not be identified straightforwardly. Wiggles can be also seen in the spectrum for a small number of extra-dimensions. However, wiggles are likely to disappear as $T$ and $a$ change during the evaporation.

When the rotation velocity is high, the deviation from the thermal spectrum is much clearer. As a novel signature, we find that the spectrum is sharply cut off at highfrequencies for rapid rotation. This new signature may survive even after we take into account the superposition of spectra along the evolutionary track of an evaporating micro black hole. This highly spinning regime is realized for $a>a_{\text {crit }}$.

In Fig. 3, the angular distribution of negative helicity particles is displayed for various parameters, setting $\omega$ to a representative frequency $\bar{\omega}$. The value $\bar{\omega}$ is chosen by requiring that the fraction of particles emitted with frequency below $\bar{\omega}, N(\bar{\omega})=\int_{0}^{\bar{\omega}} d N$, to be 0.5 .

The emission turns out to be suppressed in the direction anti-parallel to the black hole angular momentum. For rapid rotation, the particles tend to be emitted towards the equatorial plane. This concentration in the rapidly rotating case can also be seen in the helicity independent angular distribution [26]. The emission around both poles looks suppressed, but the observed apparent suppression is simply caused by the large enhancement of emission in the directions close to the equatorial plane. The asymmetry in the helicity dependent angular distribution is visible even for relatively slow rotation and becomes evident as $a$ increases. Note that, for very fast rotation, the concentration of the emitted particles toward the equatorial plane, observed in the angular distribution, may affect the features of cosmic ray air showers mediated by black holes.

For slow (rapid) rotation, the asymmetry decreases (increases) as the number of extra-dimensions $n$ grows. This tendency may be used as an indicator to discriminate scenarios with different number of extra-dimensions. It is also worth mentioning that for $a / a_{\text {crit }}$ fixed the peak

\begin{tabular}{|c|c|c|c||c|c|c|c|}
\hline$a / a_{\max }$ & 0.3 & 0.5 & 0.7 & $a / a_{\text {crit }}$ & 0.3 & 0.5 & 0.7 \\
\hline$n=2$ & 18.20 & 15.17 & 9.47 & $n=2$ & 20.68 & 16.20 & 13.17 \\
\hline$n=3$ & 19.93 & 13.43 & 8.19 & $n=3$ & 25.47 & 19.32 & 15.34 \\
\hline$n=4$ & 20.03 & 10.97 & 7.50 & $n=4$ & 29.84 & 21.75 & 17.14 \\
\hline
\end{tabular}

TABLE I: Estimate of $\delta$ in degrees for the curves of fig. 3.

position of the helicity dependent angular distribution is almost independent of $n$ as shown in upper panel, Fig. 3.

Statistics. If we can align the direction of the axis of rotation of the black hole for various events even approximately, we can collectively use the experimental data to achieve high statistics for the angular distribution of emitted particles. The LHC may allow to perform such measurements.

In the following, we will provide an estimation of the error in the determination of the axis of rotation, assuming that $N$ particles are emitted per black hole. Let $P(\Omega)$ be the angular distribution of the emitted particles. We expand it in terms of Legendre polynomials as $P(\Omega)=\sum C_{l} P_{l}(\cos \theta)$, and consider the identification of the direction of angular momentum based on $l=1$ (dipole) and $l=2$ (quadrupole) moments. For the dipole and quadrupole estimators, respectively, the errors in the estimated direction, $\delta_{d}$ and $\delta_{q}$, can be evaluated as $\delta_{d}^{2}=\frac{(1-\zeta)}{N C_{1}^{2}}$ and $\delta_{q}^{2}=\frac{4 \zeta(1-\zeta)}{N(3 \zeta-1)}$, with $\zeta=C_{0} / 3+2 C_{2} / 15$. Combining the dipole and quadrupole estimators, the $1 \sigma$ error in total can be reduced to $\delta=\left(\delta_{d}^{-2}+\delta_{q}^{-2}\right)^{-1 / 2}$. Assuming that the angular distribution shown in Fig. 3 , the error $\delta$ in degrees for $N=100$ is summarized in Table (1. Here we restricted our consideration to the dipole and quadrupole estimators, but more sophisticated statistical analyses may reduce the error.

Discussions. In the collision of two particles at transplankian energy, a rotating black hole is expected to form and decay. We studied possible signatures of rotation of such black holes in departure of the energy spectra from the thermal profile, and in the features of the helicity dependent angular distributions.

Black hole thermodynamics seems to suggest the presence of an instability for $a \geq a_{\text {crit }}$. This critical value $a_{c r i t}$ is smaller than the maximal value $a_{\max }$ allowed by the kinematical requirement of formation of a black hole in the collision of two particles. As far as $a \leq a_{\text {crit }}$, the shape of the energy spectrum is almost independent of $n$. The largest dependence on $n$ will appear in the effective temperature as shown in Figs. 2. However, this $n$-dependence must be interpreted with caution. When the ratio $a / a_{\text {crit }}$ is fixed, the enhancement of the effective temperature is larger for a smaller number of extradimensions. While the tendency is completely opposite if the ratio $a / a_{\max }$ is fixed. Hence, under the situation in which the true maximum value of $a$ is unknown, it is rather difficult to extract the information about the number of extra-dimensions without changing the energy of colliding particles [5]. 

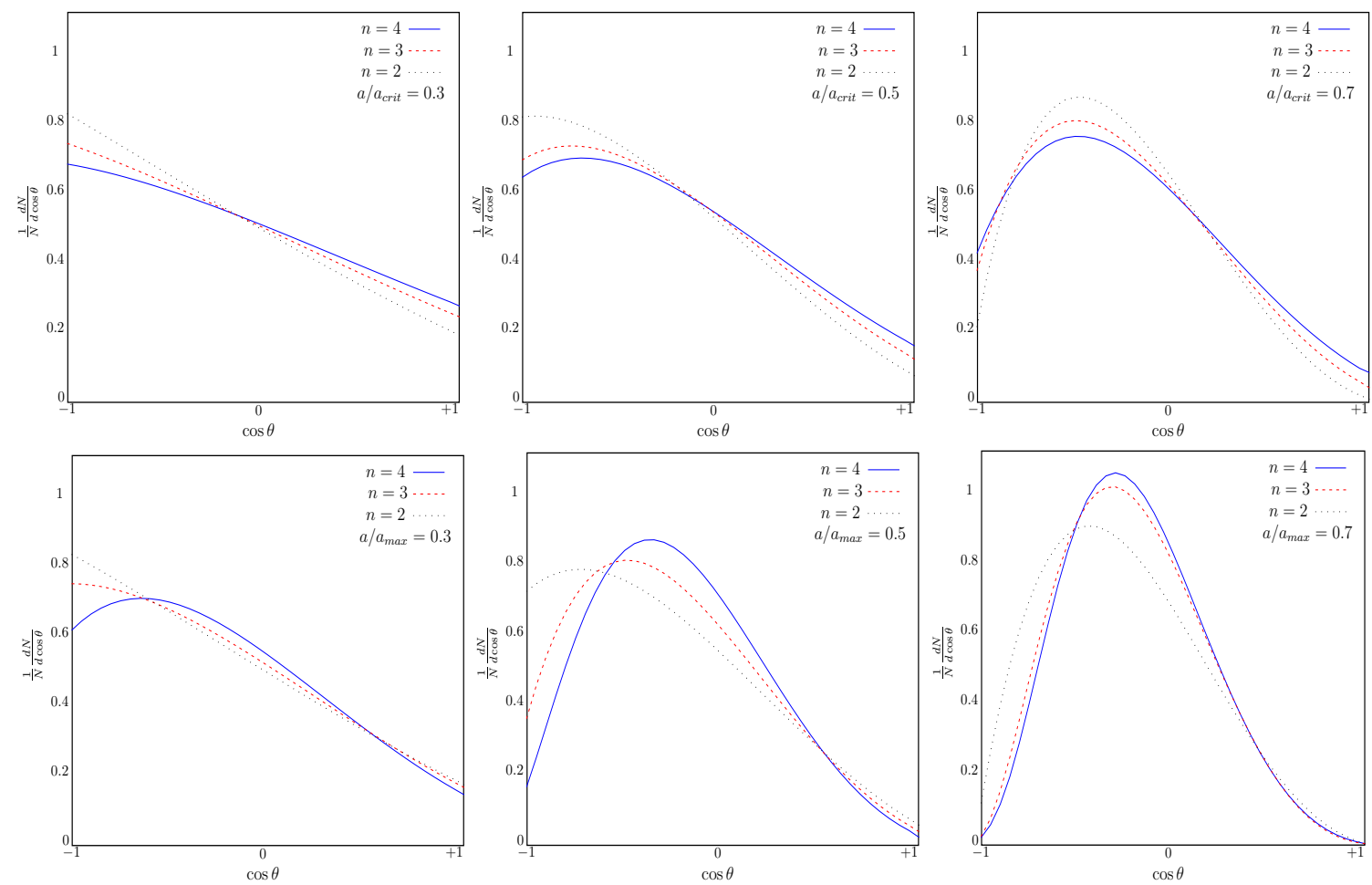

FIG. 3: The angular distribution of emitted negative helicity fermions. In each plot the cases with $n=2,3$ and 4 are shown simultaneously. The reference values of the rotation parameter are $a_{\text {crit }}$ and $a_{\max }$ in the upper and lower panels, respectively. The rotation parameter $a$ is set (left to right) to $30 \%, 50 \%, 70 \%$ of the reference value.

The peak position of the helicity dependent angular distribution may give a valuable information, because it seems to be a good indicator of $a / a_{\text {crit }}$ (or $a$ itself since $a_{\text {crit }}$ is always close to 1 ). Moreover, the amplitude of the anisotropy depends on the number of extra-dimensions. Hence, measuring the helicity dependent angular distribution may provide a very important signature to extract the value of $n$. To develop analysis of this kind based on experiment, we need to coherently accumulate data from many events. For this purpose, it is necessary to identify the rotation axis of the formed black hole for each event. We have demonstrated that this identification is marginally possible if we can detect $O(100)$ particles.

\section{Acknowledgments}

We acknowledge the support of the JSPS through Grants Nos. 19GS0219, 20740133, 17340075, 19540285 and 18204024, and Grant-in-Aid for the Global COE Program "The Next Generation of Physics, Spun from Universality and Emergence" from the Ministry of Education, Culture, Sports, Science and Technology (MEXT) of Japan. We thank Y. Sendouda for help with the numerics.
[1] N. Arkani-Hamed, S. Dimopoulos, G. Dvali, Phys. Lett. B429 (1998) 263.

[2] I. Antoniadis, N. Arkani-Hamed, S. Dimopoulos and G. Dvali, Phys. Lett. B436 (1998) 257.

[3] L. Randall, R. Sundrum, Phys. Rev. Lett. 83 (1999) 3370 .

[4] T. Banks, W. Fischler, hep-th/9906038

[5] S. Dimopoulos, G. Landsberg, Phys. Rev. Lett. 87 (2001) 161602.

[6] S. B. Giddings, S. Thomas, Phys. Rev. D65 (2002) 056010.

[7] N. Kaloper, J. Terning, Gen. Rel. Grav. 39 (2007) 1525.

[8] P.C. Argyres, S. Dimopoulos, J. March-Russell, Phys.
Lett. B441 (1998) 96.

[9] J.L. Feng, A.D. Shapere, Phys. Rev. Lett. 88 (2002) 021303.

[10] P. Kanti, arXiv:0802.2218.

[11] P. Draggiotis, M. Masip, I. Mastromatteo, JCAP 014 (2008) 0807.

[12] D.M. Eardley, S.B. Giddings, Phys. Rev. D66 (2002) 044011.

[13] H. Yoshino, Y. Nambu, Phys. Rev. D66 (2002) 065004; Phys. Rev. D67 (2003) 024009.

[14] H. Yoshino, V. Rychkov, Phys. Rev. D71 (2005) 104028.

[15] U. Sperhake, V. Cardoso, F. Pretorius, E. Berti, J.A. Gonzalez, arXiv:0806.1738 
[16] P. Kanti, Int. J. Mod. Phys. A19 (2004) 4899

[17] R. Emparan, G.T. Horowitz, R.C. Myers, Phys. Rev. Lett. 85 (2000) 499.

[18] V. Cardoso, M. Cavaglia, L. Gualtieri, Phys. Rev. Lett. 96 (2006) 071301.

[19] V.P. Frolov, D. Stojkovic, Phys. Rev. D67 (2003) 084004.

[20] D. Ida, K. Oda, S.C. Park, Phys. Rev. D67 (2003) 064025 ; erratum ibid. D69 (2004) 049901.

[21] G. Duffy, C. Harris, P. Kanti, E. Winstanley, JHEP 09 (2005) 049.

[22] C. Harris, P. Kanti, Phys. Lett. B633 (2006) 106.

[23] D. Ida, K. Oda, S.C. Park, Phys. Rev. D71 (2005) 104039.

[24] M. Casals, P. Kanti, E. Winstanley, JHEP 02 (2006) 051.
[25] D. Ida, K. Oda, S.C. Park, Phys. Rev. D73 (2006) 124022.

[26] M. Casals, S. Dolan, P. Kanti, E. Winstanley, JHEP 03 (2007) 019.

[27] R. C. Myers and M. J. Perry, Ann. Phys. 172 (1986) 304.

[28] W. Unruh, Phys. Rev. Lett. 31 (1973) 1265; Phys. Rev. D10 (1974) 3194.

[29] A. Vilenkin, Phys. Rev. Lett. 41 (1978) 1575; erratum ibid. 42 (1979) 195.

[30] W.H. Press, S.A. Teukolsky, The Astrophysical J. 185 (1973) 649.

[31] D. Page, Phys. Rev. D14 (1976) 3260.

[32] R. Emparan, R.C. Myers, JHEP 09 (2003) 025. 